Physics Vol. 3, No. 1, pp. 37-45, 1967. Physics Publishing Co. Printed in Great Britain

\title{
QUASI-ELASTIC SCATTERING OF NEUTRONS BY DILUTE POLYMER SOLUTIONS: I. FREE-DRAINING LIMIT
}

\author{
P. - G. de GENNES \\ Laboratoire de Physique des Solides associé au C.N.R.S. \\ Faculté des Sciences d'ORSAY - 91 - France \\ ( Received 20 June 1966 )
}

\begin{abstract}
In the so-called "free-draining limit", successive units of a long molecule equalise their average orientation by a diffusion process along the chain. For low values of the momentum transfer $\hbar \mathbf{q}$ and of the energy transfer $\hbar \omega$ the dynamical form factor $S(\mathbf{q} \omega)$ for neutron scattering is controlled by this effect, and is independent of the vibrational spectrum. In this regime, we show that the frequency width $\Delta \omega_{q}$ of $S(\mathbf{q} \omega)$ is small and proportional to $q^{4}$. The unusual $q^{4}$ law is related to the fact that, in a time $t$, a signal travels a distance $d \sim \sqrt{t}$ along the chain, but the corresponding distance in space is only of order $d^{1 / 2}$ for a coiled polymer. On the other hand, if the chain is stretched this argument breaks down and the width $\Delta \omega_{q}$ for coherent scattering is predicted to increase.
\end{abstract}

The existing experiments on neutron inelastic scattering by polymers [1] have been concerned mainly with the high frequency part of the spectrum $\left(\omega \sim \omega_{\max } \sim 10^{14} \mathrm{sec}^{-1}\right)$. The corresponding short wavelength vibrations and viscous motions are then characteristic of very small sections of the chain [2]. In order to achieve situations where the length of the molecule has important effects on the motion, one should concentrate on experiments where the momentum transfer $\hbar q$ of the neutron and the energy transfer $\hbar \omega$ are small [3]. In this limit the energy spectrum of the neutrons scattered by the molecule (at fixed $q$ ) will consist of a quasi-elastic peak (width $\left.\Delta \omega_{q} \ll \omega_{\max }\right)$ plus a weak background extending up to $\omega \cong \omega_{\max } .0 u r$ aim is to discuss the structure of this quasi-elastic peak. The slow motions of a long molecule in a solvent have been discussed by Rouse (4a) and by Zimm (4b). Rouse considered only the so-called "free-draining limit", where all hydrodynamic effects coupling distant segments are neglected. Zimm has included these effects. In the present paper we restrict our attention to the Rouse model: this corresponds to an extreme case, which is probably not often realised in practice, but it displays some rather interesting geometrical properties. The consequences of the long-range hydrodynamic interactions (leading to a very different $q$ dependence of $\Delta \omega_{q}$ ) will be taken up in a second paper.

\section{Fundamental Equations for the Free-draining Limit}

The long chain is divided into $N$ subunits, marked by the points $\mathbf{r}_{0}, \mathbf{r}_{1}, \ldots, \mathbf{r}_{N}$. The intervals are $\mathbf{a}_{n}=\mathbf{r}_{n+1}-\mathbf{r}_{n}$. In the absence of external forces the orientations of successive units measured at the same time are assumed independent [5].

$$
<\mathbf{a}_{n}(t) \cdot \mathbf{a}_{m}(t)>=\sigma^{2} \delta_{n m}
$$


In a solvent at rest, the equation of irreversible motion is of the form

$$
\frac{\partial \mathbf{r}_{n}}{\partial t}=-\sum_{m} B_{n m} \frac{\partial G}{\partial \mathbf{r}_{n}}
$$

where $B_{n m}$ are coefficients and $G$ is the thermodynamic potential for fixed external forces. The crucial assumption of Rouse (4a) is to assume that $B_{m n}$ is a rapidly decreasing function of $|m-n|$. Then, by a suitable redefinition of the submolecules, one can always reduce the problem to the case $B_{m n}=B \delta_{m n}$. In particular, for $O$ applied forces, $G$ contains only an entropy contribution. For small distortions

$$
G=\frac{3 k_{B} T}{2 \sigma^{2}} \sum_{n} \mathbf{a}_{n}^{2}+\text { const. }
$$

This leads to [6]:

$$
\begin{gathered}
\frac{\partial \mathbf{a}_{n}}{\partial t}=W\left(\mathbf{a}_{n+1}-2 \mathbf{a}_{n}+\mathbf{a}_{n-1}\right. \\
W=\frac{3 k_{B} T B}{\sigma^{2}}
\end{gathered}
$$

From equation (4) we derive the basic time dependent correlation functions

$$
\left\langle\mathbf{a}_{n}(0) \cdot \mathbf{a}_{m}(t)>=\sigma^{2} G_{m n}(t)\right.
$$

where $G_{m n}(t)$ is the Green's function

$$
G_{n m}(t)=\frac{1}{2 \pi} \int_{-\pi}^{\pi} d p e^{i p(n-m)} e^{-2 W(1-\cos p)|t|}
$$

We consider only the limit of very long chains and the above $G_{m n}$ applies for this case. Note that $G_{n m}(0)=\delta_{n m}$ as required by equation (1).

\section{Incoherent Scat tering}

This is of particular interest, since most polymers contain hydrogen and the incoherent cross section of $H$ is very large. The dynamical form-factor for this case is [3]

$$
\begin{aligned}
& S(\mathbf{q}, \omega)=\frac{1}{2 \pi} \int d t S(\mathbf{q}, t) e^{i \omega t} \\
& S(\mathbf{q}, t)=\left\langle e^{-i \mathbf{q} \cdot \mathbf{r}_{n}(0)} e^{i \mathbf{q} \cdot \mathbf{r}_{n}(t)}\right\rangle
\end{aligned}
$$

For $t \gg W^{-1}\left(W^{-1}\right.$ being a typical correlation time), $\mathbf{r}_{n}(t)-\mathbf{r}_{n}(0)$ is a sum of a large number of independent contributions and has a gaussian distribution. Then

$$
S(\mathbf{q}, t)=e^{-\frac{1}{2} q^{2}\left\langle\left[x_{n}(0)-x_{n}(t)\right]^{2}\right\rangle}
$$

where $x_{n}$ is the projection of $r_{n}$ along q. For an infinite chain we can write 


$$
\begin{aligned}
& \mathbf{r}_{n}=\sum_{m=-\infty}^{m=n-1} \mathbf{a}_{m} \\
& \frac{1}{2}<\left[x_{n}(0)-x_{n}(t)\right]^{2}>=\frac{1}{3}<\mathbf{r}(0) \cdot\left[\mathbf{r}_{n}(0)-\mathbf{r}_{n}(t)\right]> \\
& =\frac{1}{3} \sum_{m<n} \sum_{m^{\prime}<n}<\mathbf{a}_{m}(o)\left[\mathbf{a}_{m}^{\prime}(o)-\mathbf{a}_{m}^{\prime}(t)\right]> \\
& =\frac{\sigma^{2}}{3} \int_{-\pi}^{\pi} \frac{d p}{2 \pi} \sum_{m<n} \sum_{m^{\circ}<n} e^{i p\left(m-m^{\prime}\right)}\left[1-e^{-2 W(1-\cos p) t}\right]
\end{aligned}
$$

The sum $\sum_{m m^{\prime}}$ is easily carried out:

$$
\sum_{m<n} \sum_{m^{\prime}<n} e^{i p\left(m-m^{\prime}\right)}=\frac{1}{2(1-\cos p)}
$$

We are only interested in the region $W_{t} \gg 1$ and the integrand in $\int d p$ is non-vanishing only for small $p$. Then

$$
\begin{aligned}
\frac{1}{2}<\left[x_{n}(0)-x_{n}(t)\right]^{2}> & \rightarrow \frac{\sigma^{2}}{6 \pi} \int_{-\infty}^{\infty} \frac{d p}{p^{2}}\left(1-e^{\left.-W^{2}|t|\right)}\right. \\
& =\frac{\sigma^{2}}{3} \sqrt{\frac{W|t|}{\pi}}
\end{aligned}
$$

The unusual square root dependence of equation (10) (can be understood as follows: in time $t$, a signal starting from one given submolecule will reach $Q \sim \sqrt{W t}$ neighboring submolecules. But since the polymer is coiled, the distance in space corresponding to this length is $\Delta x \sim \sigma \sqrt{Q}$. Thus $\Delta x^{2} \sim \sigma^{2} \sqrt{W t}$. It is of interest to verify that equation (10) is independent of the size chosen to define the submolecules. Introducing the diffusion coefficient for the center of gravity of the total molecule $D=B k_{B} T / N=(1 / 3 N) W \sigma^{2}$ and the total mean square extension $R^{2}=N \sigma^{2}$, we may write in fact [7] $\sigma^{2} \sqrt{W t}=R \sqrt{3 D t}$.

The quantity which is directly measured is $S(\mathbf{q} \omega)$ as defined by (7). It is expressible in terms of the Fresnel integrals $C(x), S(x)$ [8].

$$
\begin{aligned}
S(\mathbf{q}, \omega) & =\left(\frac{3}{q^{2} \sigma^{2}}\right)^{2} \frac{1}{W} Z(\rho) \\
Z(\rho) & =\sqrt{\frac{\pi}{2}} \rho^{-3 / 2}\left\{\left[\frac{1}{2}-S\left(\frac{1}{4 \rho}\right)\right] \sin \frac{1}{4 p}+\left[\frac{1}{2}-C\left(\frac{1}{4 p}\right)\right] \cos \frac{1}{4 p}\right\} \\
\rho & =\frac{\pi \omega}{W}\left(\frac{3}{q^{2} \sigma^{2}}\right)^{2}
\end{aligned}
$$




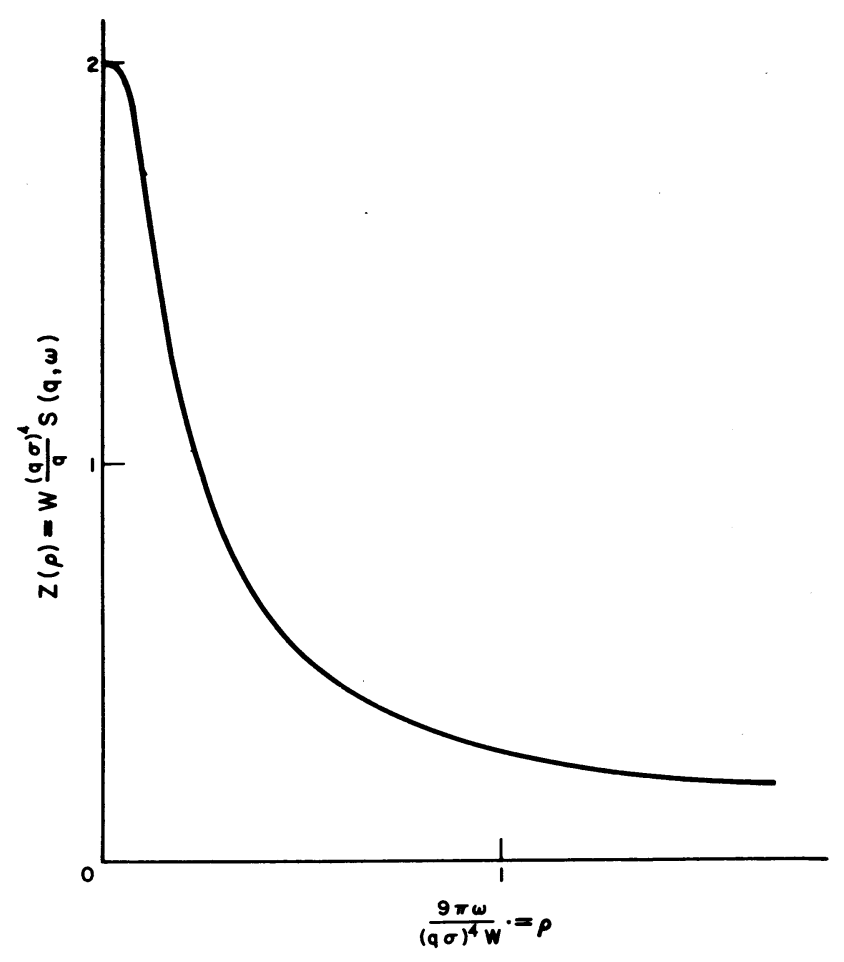

FIGURE 1

The dynamical form factor for incoherent scattering at fixed (small) momentum transfer $\hbar q$, as a function of the energy transfer $\hbar \omega$.

A plot of $Z(\rho)$ is given on Fig. 1. The half-width at half-maximum $\Delta p$ of $Z(p)$ corresponds roughly to $\Delta p=1 / 4$ or

$$
\Delta \omega q \sim 10^{-2} W \sigma^{4} q^{4} \cong 3 \times 10^{-2} D R^{2} q^{4}
$$

The range of validity of this formula is defined by

$$
q R \gg 1 \gg q b
$$

where $b$ is an atomic distance (more precisely, for a simple chain with fixed valence angle $\theta$ and interatomic distance $a, b=a(1+\cos \theta / 1-\cos \theta))$.

There are at least two difficulties to overcome if one wishes to study the quasi-elastic peak:

(a) the energy width $\hbar \Delta \omega q$ predicted by (12) is very small: taking $\sigma q=1 / 2$ and $W=10^{14}$ $\sec ^{-1}$ (a rather high value corresponding to a solvent of low viscosity), we get $\hbar \Delta \omega q \sim 1^{\circ} \mathrm{K}$;

(b) the experiment should be done on a relatively dilute solution, but the "noise" coming from the solvent molecules must be low: in particular, the solvent should not contain hydrogen atoms. 


\section{Coherent Scattering}

This situation may be of interest if the coherent scattering amplitude (per unit volume) for the polymer is much larger than the amplitude for the solvent. The correlation function to be studied is:

$$
S_{\mathrm{coh}}(\mathbf{q} t)=\sum_{i j} a_{i} a_{j}<e^{i \mathbf{q} \cdot\left[\mathbf{r}_{i}(t)-\mathbf{r}_{j}(o)\right]}>
$$

where the sum $\Sigma_{i j}$ runs on all atoms in the long molecule, and $a_{i}, a_{j}$ are characteristic amplitudes. For an atom $i$ belonging to the $n$th submolecule we separate

$$
\mathbf{r}_{i}=\mathbf{r}_{n}+\rho_{i}
$$

At the time of interest ( $\left.W_{t} \gg 1\right)$ the vectors $\rho_{i}(t)$ and $\rho_{j}(o)$ are uncorrelated between themselves and uncorrelated to the $\mathbf{r}_{n}{ }^{\prime} \mathbf{s}$. Then, for two atoms $i$ and $j$ belonging respectively to the submolecules $n$ and $m$ ( $n$ and $m$ being different or equal)

$$
e^{i \boldsymbol{q} \cdot\left[\mathbf{r}_{i}(t)-\mathbf{r}_{j}(0)\right]}=\left\langle e^{i \boldsymbol{q} \cdot \boldsymbol{P}_{i}}\right\rangle\left\langle e^{-i \boldsymbol{q} \cdot \boldsymbol{P}_{j}}\right\rangle\left\langle e^{i \boldsymbol{q} \cdot\left[\mathbf{r}_{n}(t)-\mathbf{r}_{m}(0)\right]}\right\rangle
$$

Introduce a form factor

$$
F(\mathbf{q})=\sum_{i} a_{i}<e^{i \boldsymbol{q} \cdot \boldsymbol{P}_{i}}>
$$

where the sum is carried over the atoms in one submolecule. Then

$$
S_{c \circ h}(\mathbf{q} t)=|F(q)|^{2} \sum_{n m}<e^{i \mathbf{q} \cdot\left[\mathbf{r}_{n}(t)-\mathbf{r}_{m}(0)\right]}>
$$

For $W_{t} \gg 1$ (and for the distance scale specified by $q b \ll 1$ ) we can again assume that the distribution of $\mathbf{r}_{n}(t)-\mathbf{r}_{m}(o)$ is gaussian and write

$$
S_{\text {coh }}(\mathbf{q} t)=|F(q)|^{2} \sum_{n m} e^{-1 / 2 q^{2}\left\langle\left[x_{n}(t)-x_{m}(0)\right]^{2}\right\rangle}
$$

Expressing the $\mathbf{r}_{n}$ 's in terms of $\mathbf{a}^{\prime} \mathbf{s}$ and using equation (5), we arrive at

$$
\begin{aligned}
\frac{1}{2}<\left[x_{n}(t)-x_{n+s}(o)\right]^{2}> & =\frac{\sigma^{2}}{6 \pi} \int_{-\infty}^{\infty} \frac{d p}{p^{2}}\left(1-e^{-W p^{2}|t|} \cos p s\right) \\
& =\frac{\sigma^{2}}{6}\left[|s|+2 \sqrt{\frac{W t}{\pi}} g\left(\frac{s^{2}}{4 W|t|}\right)\right] \\
g(u) & =\int_{1}^{\infty} \frac{d \tau}{\tau^{2}} e^{-u \tau^{2}}
\end{aligned}
$$

The double sum $\Sigma_{n m}$ in equation (17) then reduces to $N \Sigma_{s}$ and, for the long distance limit of 
interest $(q \sigma \ll 1) \Sigma_{s}$ may be replaced by $\int d s$. Setting

$$
\begin{aligned}
& s=2 \sqrt{W|t|} \lambda \\
& \theta=\frac{q^{2} s^{2}}{3}(W|t|)^{1 / 2}
\end{aligned}
$$

we obtain:

$$
\begin{aligned}
& \frac{S_{\mathrm{coh}}(\mathbf{q} t)}{S_{\mathrm{coh}}(\mathbf{q}, t=0)}=\theta \int_{0}^{\infty} d \lambda e^{-\theta\left[\lambda+(1 / \sqrt{\pi}) g\left(\lambda^{2}\right)\right]} \\
& S_{\mathrm{coh}}(\mathbf{q}, t=0)=N|F(q)|^{2} \frac{12}{\sigma^{2} q^{2}}=\int_{\infty}^{\infty} d \omega S_{\mathrm{coh}}(\mathbf{q} \omega)
\end{aligned}
$$

Equations (21) and (22) deserve a few comments: (a) Equation (22) displays the well-known $1 / q^{2}$ singularity of the total scattering power at small angles. This large intensity may be useful to separate background effects and represents a definite advantage of coherent versus incoherent scattering. (b) Equation (21) shows that $S_{\text {coh }}(\mathbf{q} t)$ depends on only one particular combination of $q$ and $t$, namely the variable $\theta$ defined in equation (20). Just as in the incoherent case we can verify that $\theta$ is independent of the size chosen to define the submolecules.

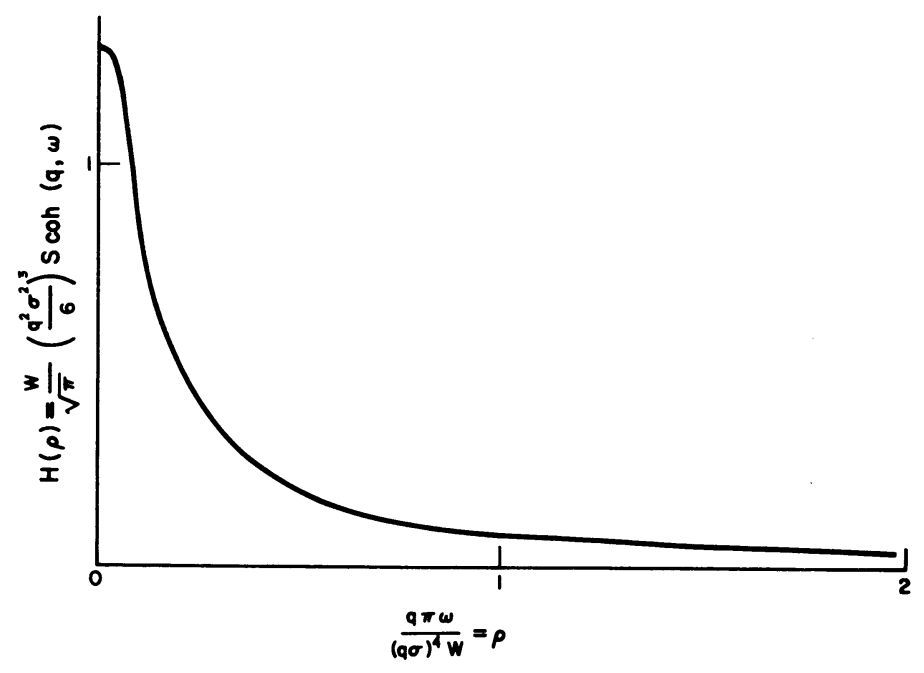

FIGURE 2 .

The dynamical form factor for coherent scattering for a long molecule (under $O$ applied forces) at fixed (small) momentum transfer $\hbar q$, as a function of the energy transfer $\hbar \omega$.

Finally, the calculation of the energy distribution $S_{\text {coh }}(q \omega)$ (i.e. the Fourier transform of equation (21)) requires some numerical work. The results are shown on Fig. 2. The general aspect is not very different from what was obtained for incoherent scattering. The half-width at half-maximum is 


$$
\Delta \omega_{q} \cong 2 \times 10^{-2} D R^{2} q^{4}
$$

\section{Effect of Stretching the Molecule}

We now show that if the molecule is stretched, the frequency width $\Delta \omega_{q}$ expected for coherent scattering (with $q$ along the direction of elongation) can increase significantly, $\Delta \omega_{q}$ being now proportional to $q^{2}$ instead of $q^{4}$. In practice, the elongation might be achieved either on a connected (but still rather dilute) polymer network (swollen rubber) or by electric fields on bolar molecules.

At equilibrium, the average orientation of each submolecule is now nonzero:

$$
<\mathbf{a}_{n}>=\mathbf{l} \neq 0
$$

In the following we shall consider only weak elongations $(l<\sigma)$. Then the Rouse equation (4) still applies [9] and the basic correlation functions are

$$
<a_{n \alpha}(0) a_{m \beta}(t)>=\left(\frac{1}{3} \sigma^{2} \delta_{\alpha \beta}-l_{\alpha} l_{\beta}\right) G_{n m}(t)+l_{\alpha} l_{\beta}
$$

There $\alpha, \beta=x, y, z$ and $G_{n m}$ is always defined by (6).

For incoherent scattering this modification has only minor effects. But for coherent scattering, we obtain, instead of equations $(17,18)$ :

$$
\left.S(\mathbf{q} t)=\sum_{s=-\infty}^{s=\infty} e^{i s \mathbf{q} \cdot 1} e^{-\left(q^{2} \sigma^{2} / \sigma\right)[|s|+2 \sqrt{W t / \pi}} g\left(S^{2} / 4 W t\right)\right]
$$

If $q \cdot \mathbf{l} \ll q^{2} \sigma^{2}$ equation (24) goes back into equation (18). But if $q \cdot l \gg q^{2} \sigma^{2}$ (namely if $\mathbf{I}$ is parallel to $\mathbf{l}$, and $\left.q<l / \sigma^{2}\right)$, the behavior is profoundly different. The times of interest are then $t \sim\left(W q^{2} l^{2}\right)^{-1}$. The last exponential in equation (24) can then be expanded

$$
e^{-q^{2} \sigma^{2} / 3 \sqrt{K^{2} t / \pi} g}=1-\frac{q^{2} \sigma^{2}}{3} \sqrt{W t / \pi} g
$$

and after some manipulation this leads to

$$
S(\mathbf{q} t)=\frac{q^{2} \sigma^{2}}{3(\mathbf{q} \cdot \mathbf{1})^{2}} e^{-W t(\mathbf{q} \cdot \mathbf{1})^{2}}
$$

The Fourier transform $S_{\text {coh }}(\mathbf{q} \omega)$ is then a Lorentz curve of width

$$
\left(\Delta \omega_{q}\right)_{\text {stretched }}=W(\mathbf{q} \cdot \mathbf{1})^{2} \quad\left(\mathbf{q} \cdot \mathbf{l} \gg q^{2} \sigma^{2}\right)
$$

Note that in this case $\Delta \omega_{q}$ is larger than the value at rest $\left(\sim W q^{4} \sigma^{4}\right)$. Thus in certain conditions an elongation should cause a broadening of the quasi-elastic peak. 


\section{Concluding Remarks}

Our discussion was based on the Rouse assumption: the drift velocity $\frac{d}{d t} \mathbf{r}_{n}$ is proportional to the local curvature $a_{n}-a_{n+1}$. This assumption is interesting because of it's simplicity, and it has led us to results which have a simple geometrical interpretation. But it is not realistic: in actual fact $\frac{d}{d t} \mathbf{r}_{n}$ depends on the configuration of submolecules ( $m$ ) very remote from $(n)$ along the chain - for (at least) two reasons:

(a) The motion of $(n)$ relative to the solvent creates a backflow which reacts on $(n)$. This coupling leads to very different results $\left(\Delta \omega_{q} \sim q^{3}\right)$ and will be discussed separately.

(b) There may be some direct friction between $(n)$ and $(m)$.

Thus (and in spite of obvious experimental difficulties), neutron scattering experiments could reveal some interesting motional properties of long molecules.

\section{Acknowledgments}

The author is greatly indebted to J.P. Hurault for assistance in the calculations of section 3 , and to $S$. Alexander for a discussion of long range interactions.

\section{References}

1. W.M. MYERS, G.C. SUMMERFIELD and J.S. KING, Symposium on Inelastic Scattering of Neutrons, edited by Brookhaven National Laboratory, p. 126 (1965). W.L. WHITTEMORE, ibid., p. 131.

2. Theoretical attempts to describe the vibration spectrum include the following:

D. T. GOLDMAN and F.T. FEDERIGHI, Nucl. Sci. Eng. 16, 165 (1963).

J.U. KOPPEL and J.A. YOUNG, ibid. 21, 268 (1965).

R.A. HARRIS and J.E. HEARST, J. Chem. Phys. 44, 2595 (1966).

3. For the definition of $h \omega, h q$, and of the dynamical form factors, see L. VAN HOVE, Phys. Rev. 95, 249 (1954).

For a review of experiments and theory in liquids see for instance P.G. DE GENNES, in Inelastic Scattering of Neutrons by Solids and Liquids. International Atomic Energy Agency Vienna (1961).

4a. P.E. ROUSE, J. Chem. Phys. 21, 1272 (1953).

4b. B. H. ZIMM, J. Chem. Phys. 24, 269 (1956).

5. Excluded volume effects are neglected.

6. There is a striking analogy between the problem of a freely orienting chain, of units 
$\vec{a}_{1}, \vec{a}_{2}, \ldots, \vec{a}_{N}$, and a one-dimensional assembly of spins $\vec{S}_{1}, \ldots, \vec{S}_{N}$ coupled by exchange forces. In both cases the fundamental processes contributing to the entropy source are local motions which do not change the total vector length $\vec{S}_{1}+\ldots+\vec{S}_{N}$. From this conversation property the diffusion form of equation (4) follows immediately.

7. Note that, although $R$ and $D$ depend on the molecular weight $M$ of the polymer, the product $R \sqrt{D}$ is independent of $M$. All the properties discussed assume only that the chain is long $(q R \gg 1)$.

8. The functions $C$ and $S$ are defined as in Jahnke-Emde's Tables of Higher Functions (Teubner ed.), p. 28, Stuttgart (1960).

9. Except of course for the end points, where the contribution of the applied forces must be included. 